\title{
Titanium enrichment and strontium depletion near edge dislocation in strontium titanate $[001] /(110)$ low-angle tilt grain boundary
}

\author{
K. Takehara $\cdot$ Y. Sato $\cdot$ T. Tohei $\cdot$ N. Shibata $\cdot$ \\ Y. Ikuhara
}

Received: 29 September 2013/ Accepted: 9 January 2014/Published online: 11 March 2014

(C) The Author(s) 2014. This article is published with open access at Springerlink.com

\begin{abstract}
Dislocations are linear lattice defects in a crystalline solid. Since the unusual atomistic environment of the dislocation may greatly influence various material properties, control of the composition would offer more opportunities to obtain unique one-dimensional structures. In the present study, we have characterized the structure of dislocations in a low-angle tilt grain boundary of strontium titanate $\left(\mathrm{SrTiO}_{3}\right)$. High-spatial resolution elemental mapping by electron energy loss spectroscopy combined with scanning transmission electron microscopy has enabled visualization of the enrichment of titanium (Ti) and the depletion of strontium $(\mathrm{Sr})$ near the dislocation cores. The $\mathrm{Ti}$ enrichment and the $\mathrm{Sr}$ depletion have been observed at all of the dislocations, and the grain boundary is considered to be $\mathrm{Ti}$ excess. The extra $\mathrm{Ti}$ ions are located on the positions different from the normal perovskite lattice,
\end{abstract}

K. Takehara · Y. Sato · T. Tohei · N. Shibata · Y. Ikuhara $(\bowtie)$ Institute of Engineering Innovation, The University of Tokyo, 2-11-16, Yayoi, Bunkyo, Tokyo 113-8656, Japan

e-mail: ikuhara@sigma.t.u-tokyo.ac.jp

N. Shibata

Japan Science and Technology Agency, PRESTO,

4-1-8, Honcho, Kawaguchi, Saitama 332-0012, Japan

Y. Ikuhara

Nanostructures Research Laboratory, Japan Fine Ceramics

Center, 2-4-1, Mutsuno, Atsuta, Nagoya 456-8587, Japan

Y. Ikuhara

World Premiere Institute for Advanced Institute for Materials Research, Tohoku University, 2-1-1, Katahira, Aoba,

Sendai 980-8577, Japan

Y. Ikuhara

Center for Elements Strategy Initiative for Structure Materials, Kyoto University, Sakyo, Kyoto 606-8501, Japan suggesting that the local structure is largely reconstructed. It has been proposed that tensile strain at the dislocations may be a cause of the Ti enrichment.

\section{Introduction}

Dislocations are linear lattice defects in a crystalline solid. Atomic arrangement at the core differs considerably from that in the bulk, and there is a high strain field around a dislocation. It is expected that the unusual atomistic environments around the dislocation may greatly influence various material properties, and several attempts to functionalize dislocations themselves have been reported [1-3]. With more sophisticated ways to control dislocation structure and composition, we will have more opportunities to create unique one-dimensional structures. In many cases, dislocations are generated during mechanical deformation and crystal growth. However, it is usually difficult to control dislocation characters (i.e., the Burgers vector and the dislocation line direction). An alternative method to obtain dislocations is a fabrication of a low-angle grain boundary (GB) in a bicrystal [3-10], which offers two benefits. One is that dislocations with desirable characters can be fabricated by controlling the GB plane and the orientation relationship of adjacent crystals. The other is that a periodic array of dislocations at desired intervals can be obtained.

$\mathrm{SrTiO}_{3}$ (STO) is a prototype perovskite oxide, whose properties have been extensively studied. Dislocations in STO are believed to play a critical role in its mechanical deformation that exhibits unusual temperature dependence [11, 12]. It has also been demonstrated that application of an electrical bias switches electrical conductivity along STO dislocations from a non-metallic to a metallic state [2]. As a result of their impact on material properties, the 
Fig. 1 a STO bicrystal with $[001] /(110) 5^{\circ}$ tilt GB.

b Dislocation array expected for the bicrystal. An array of $a / 2[110]$ edge dislocations with $\sim 3 \mathrm{~nm}$ interval is expected.

The inversed " $T$ " shape, dark dotted lines, and hatches respectively show the dislocations, dislocation lines and the stacking faults (a) STO bicrystal

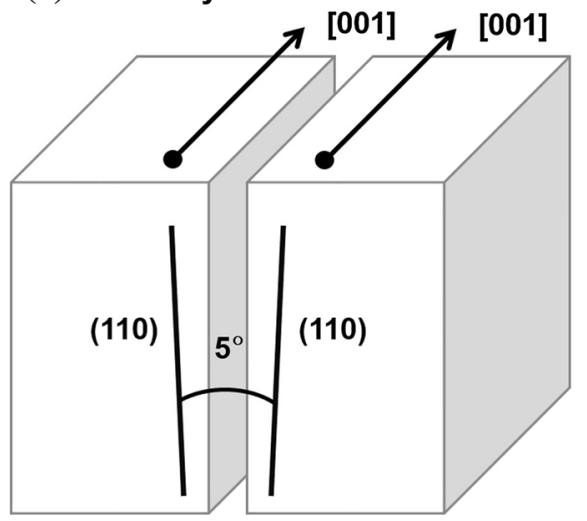

(b) Dislocation array

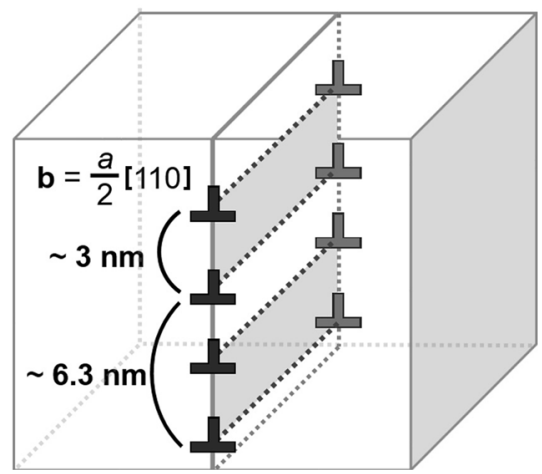

atomistic structures of the dislocations have been intensively investigated in previous studies, and the dislocations were obtained by using bicrystals in most cases [4-8]. Some of the reports suggest that the local chemical composition at the dislocation widely varies. Although most of these suggestions have been based on transmission electron microscopy (TEM) or scanning TEM (STEM) image contrast, visualization of elemental distribution around dislocations would provide better access to an understanding of this issue. Recent success in the atomic scale visualization of elemental distribution offers a greater potential for achieving this with the use of electron energy loss spectroscopy (EELS) combined with STEM [13]. In the present study, we report atomic scale imaging and spectroscopic analysis of a periodic dislocation array in a low-angle tilt GB of STO with the use of STEM imaging and STEM EELS.

\section{Experimental procedures}

STO bicrystal with a $[001] /(110) 5^{\circ}$ tilt GB (Fig. 1a, b) was fabricated by thermal diffusion bonding of two STO single crystals. First, $2.5^{\circ}$ off (110) surfaces of the single crystals (Furuuchi Chem. Co.) were polished to a mirror-like state. Then, the surface was cleaned with ethanol and propanol for removing contaminants. Subsequently, one crystal was set on the other so that the bicrystal possess $5^{\circ}$ tilt GB. Under the uniaxial load of $\sim 0.2 \mathrm{MPa}$, the two crystals were heat-treated at $700{ }^{\circ} \mathrm{C}$ for $20 \mathrm{~h}$ at the heating and cooling rate of $20^{\circ} \mathrm{C} / \mathrm{h}$ in air for bonding. Post annealing was carried out to obtain larger bonded area. Heat treatments were performed at $1000{ }^{\circ} \mathrm{C}$ for $80 \mathrm{~h}$ in total and subsequently at $700{ }^{\circ} \mathrm{C}$ for $14 \mathrm{~h}$.

Thin foils for STEM observations were prepared by a conventional method that includes mechanical polishing and argon-ion beam milling. The ion-beam milling was carried out using PIPS ${ }^{\mathrm{TM}}$ (Model 682, Gatan Inc.) with the acceleration voltage of $3.5 \mathrm{kV}$ until a hole was made. Finally, low-voltage milling was carried out with the acceleration voltage of $0.5 \mathrm{kV}$ for reducing the thickness of the irradiation damaged layers.

STEM observations were performed using an aberration-corrected STEM (ARM-200CF, JEOL Ltd.). Probeforming aperture semiangle was $24 \mathrm{mrad}$ for STEM imaging. Detection angle range for annular dark-field (ADF) images was $90-370 \mathrm{mrad}$ or $40-90 \mathrm{mrad}$. Energy dispersive X-ray spectroscopy (EDS) and EELS were performed using JED-2200 system (JEOL Ltd.) and Enfina spectrometer (Gatan Inc.), both are attached to JEM-2100F microscope (JEOL Ltd.). Probe-forming aperture semiangle was $\sim 22 \mathrm{mrad}$ and diameter of the electron probe was $\sim 0.1 \mathrm{~nm}$ for EDS and EELS. Collection angle, energy dispersion, and exposure time for EELS were $\sim 30 \mathrm{mrad}$, $0.5 \mathrm{eV} /$ channel, and $0.2 \mathrm{~s} / \mathrm{pixel}$, respectively.

Atomic column positions in STEM image were analyzed by Gaussian peak fitting. The peak fitting was carried out using our home-made software. For the fitting, twodimensional Gaussian function with the form of

$Z=Z_{0}+A \cdot \exp \left\{-\frac{1}{2}\left(\frac{x-x_{c}}{w}\right)^{2}-\frac{1}{2}\left(\frac{y-y_{c}}{w}\right)^{2}\right\}$

was used, where $Z, Z_{0}, A, w$ are total, background, peak intensities, and the peak width, and $\left(x_{c}, y_{c}\right)$ is the peak position. The variables $\left[Z, Z_{0}, A, w\right.$, and $\left.\left(x_{c}, y_{c}\right)\right]$ are optimized by the least squares method so that the squared difference between the original and the fitted intensities is iteratively converged to a minimum value. It was demonstrated that a similar procedure successfully works for analysis of STEM images [14].

\section{Results and discussion}

In the simplest model, it is expected that periodic array of $a[110]$ edge dislocations forms along the $[001] /(110) 5^{\circ}$ tilt 


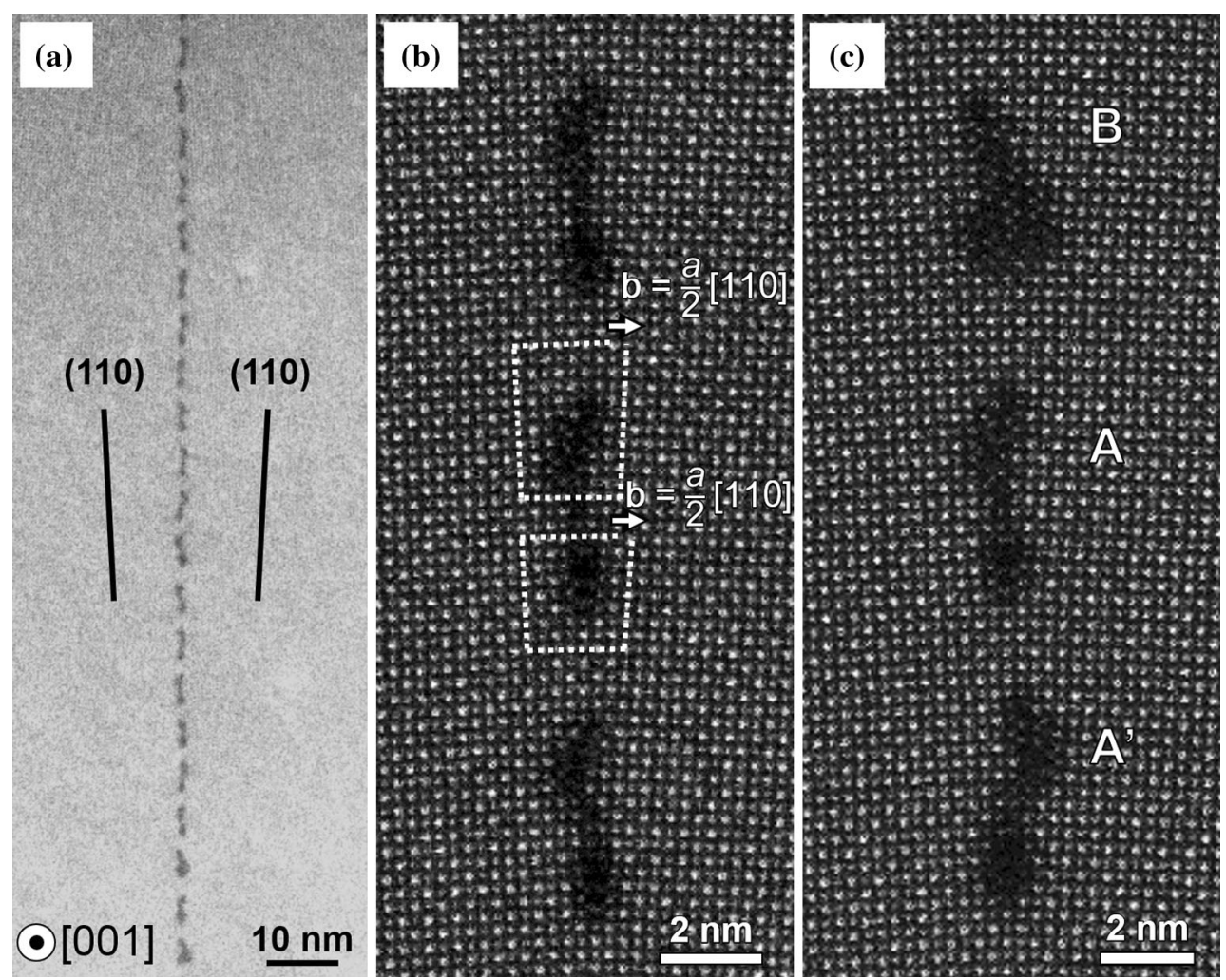

Fig. 2 Dislocation structure in the STO GB. a Low-magnification, and b, c high-magnification ADF STEM images. Dotted squares indicate Burgers circuits in $\mathbf{b}$. Dislocations of three types, $A, A^{\prime}$, and $B$ are shown in $\mathbf{c}$

GB in a STO bicrystal, where the separation distance is estimated to be $6.3 \mathrm{~nm}$ by the Frank's formula [15]. In the actual case, $a$ [110] edge dislocation is climb dissociated into two $a / 2$ [110] partial dislocations and a stacking fault where the spacing between the partials is $\sim 3 \mathrm{~nm} \mathrm{[4]}$ (Fig. 1b).

STEM imaging has confirmed that the periodic array of dislocations is formed in the bicrystal (Fig. 2a). Dislocations are observed as darker areas and pair-wise contrast observed in ADF STEM image are due to the climb dissociation of a dislocation as described below. Spacing of the dislocation pairs is about $6.3 \pm 0.3 \mathrm{~nm}$, which is consistent with the expected value. From the enlarged view, it is found that dislocations are actually dissociated into two partial dislocations (Fig. 2b). The Burgers vectors are $a / 2[110]$, where $a$ is the lattice constant of STO $(0.3905 \mathrm{~nm}$ [16]). We have observed 78 dislocations in the bicrystal. Most of them are categorized into a few major types in terms of relative locations of the partials and size of dark regions (Fig. 2c). Approximately $53 \%$ of dislocations are categorized as A type, where two partials align in the same vertical plane, and a dark region is narrow. Another $19 \%$ also has a narrow dark region, where one partial dislocation slightly shifts perpendicularly to the GB with respect to the other. Dislocations of this type are categorized as $\mathrm{A}^{\prime}$ type. Another $13 \%$ of dislocations have larger areas of dark regions particularly in the lower part, which has been categorized as B type.

The dislocation structure is further investigated in the atomic scale (Fig. 3a-c). In the ADF STEM images of STO crystal observed with [001] electron incident direction, Sr $(Z=38)$ and $\mathrm{Ti}-\mathrm{O}(Z=22$ and 8$)$ columns are observed as the brightest and second brightest spots, while O columns are not visible. This is because the ADF image exhibits atomic-number $(Z)$ dependent image contrast: The image contrast is higher for the heavier element. As mentioned above, there are darker regions near the partials. In these areas, a number of extra bright spots are recognized (Fig. 3d-f). These extra spots are located on unusual positions for a STO crystal lattice. Among the three types of dislocations, the A type has the smallest number of the extra spots. There are a few more extra spots in the $A^{\prime}$ type. On the other hand, the dark area spreads more widely in the B type. In this case, clear distinction of partial dislocations and the stacking fault is difficult. The extra spots would correspond to cations: $\mathrm{Sr}$ and/or $\mathrm{Ti}$ ions.

In order to understand the origin of the extra spots, the elemental distribution around the dislocations was mapped out by EELS. The two-dimensional maps (Fig. 4) reveal that $\mathrm{Ti}$ is enriched and $\mathrm{Sr}$ is depleted, while $\mathrm{O}$ amount is 


\section{A type $(53 \%)$}

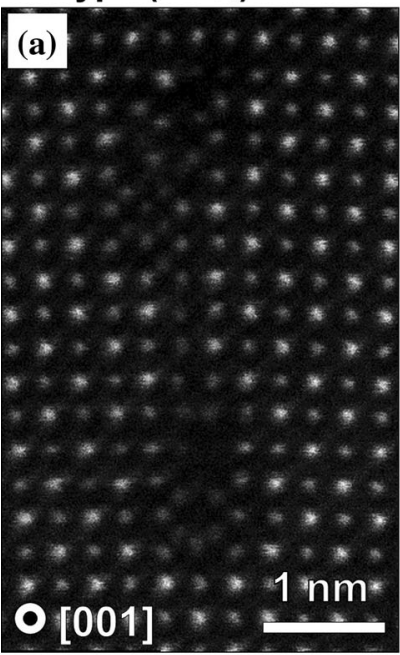

\section{A' type (19\%)}
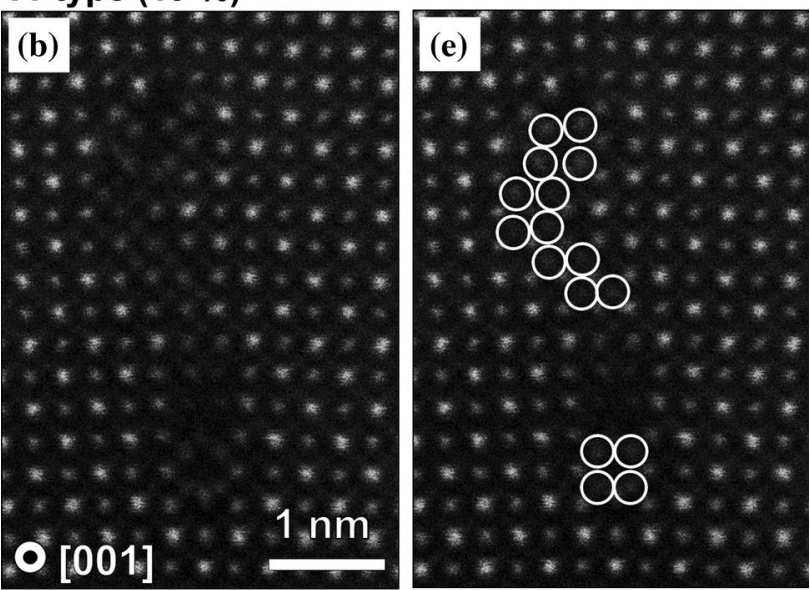

\section{B type $(13 \%)$}
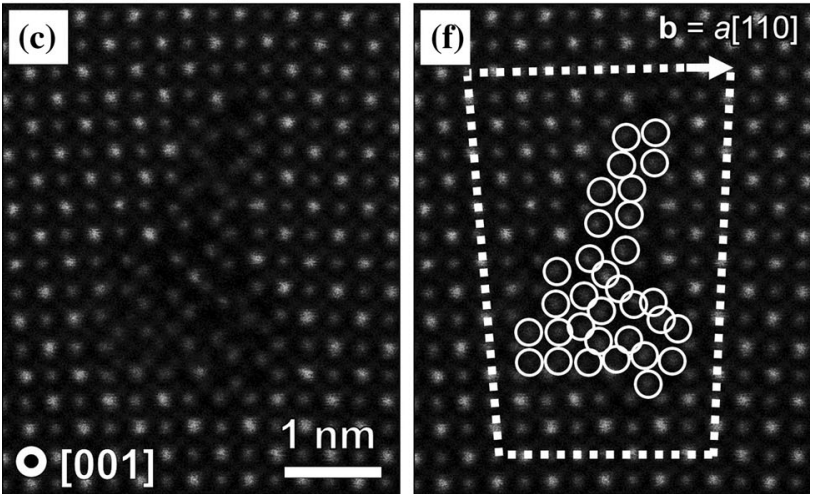

Fig. 3 Magnified ADF STEM images of the dislocations. a, $\mathbf{d}$ The A type, $\mathbf{b}, \mathbf{e}$ the $\mathrm{A}^{\prime}$ type, and $\mathbf{c}, \mathbf{f}$ the $\mathrm{B}$ type dislocations. Open circles in the right-hand side figures indicate the location of extra bright contrast. The dotted square indicates the Burgers circuit in $\mathbf{f}$

relatively unchanged near the dislocations. This tendency is common for the A (Fig. 4a-d), the A' (Fig. 4e-h), and the B (Fig. 4i-1) types. In the present study, we have measured EELS maps for about ten dislocations, and all of them

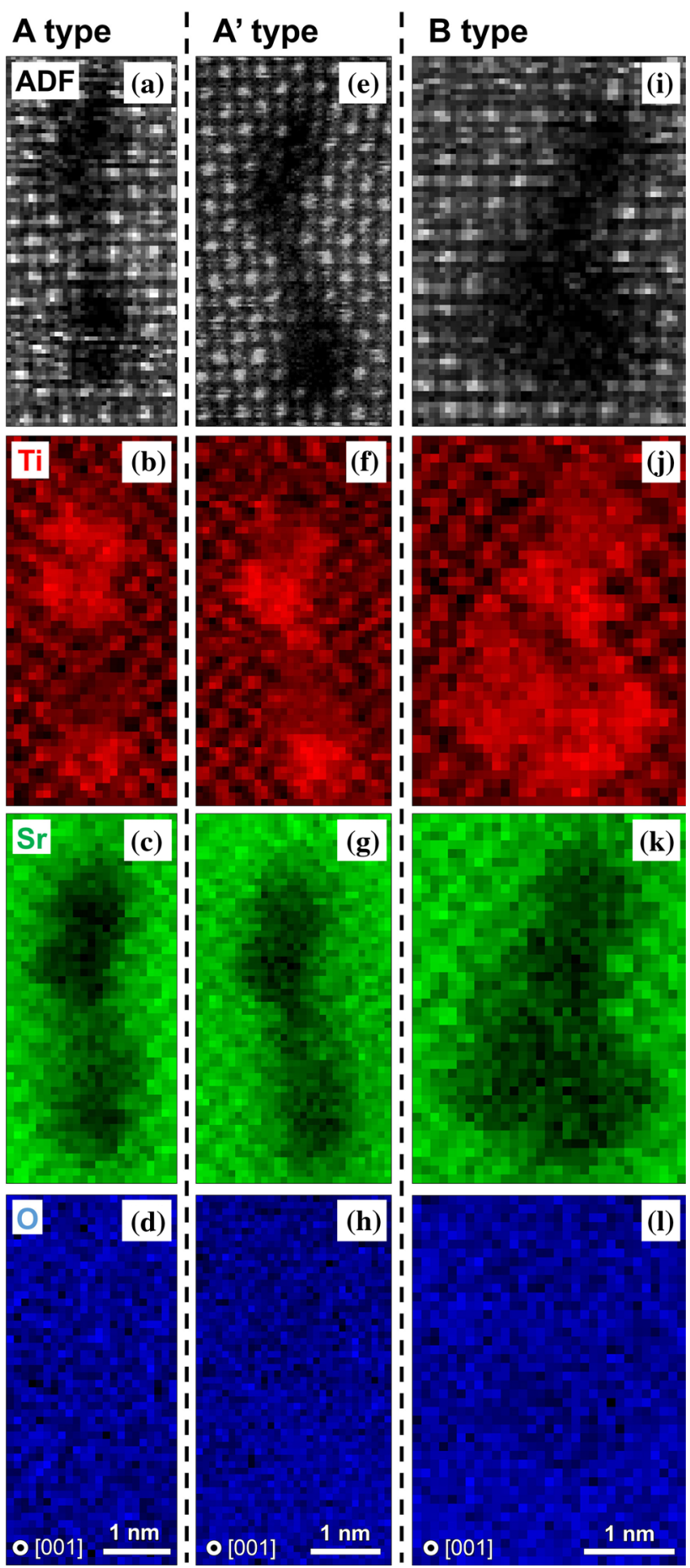

Fig. 4 EELS maps around the dislocations. a-d The A type, $\mathbf{e}-\mathbf{h}$ the $\mathrm{A}^{\prime}$ type, and $\mathbf{i}-\mathbf{l}$ the $\mathrm{B}$ type dislocations. Simultaneously recorded ADF STEM images, Ti- $\mathrm{L}_{23}$ map, $\mathrm{Sr}-\mathrm{M}_{45}$ map, and $\mathrm{O}-\mathrm{K}$ map are shown from the top to the bottom

exhibit $\mathrm{Ti}$ enrichment and $\mathrm{Sr}$ depletion. This indicates that the GB in the bicrystal is Ti rich. On the other hand, the Ti-enriched regions are located near the partials in the A and the $\mathrm{A}^{\prime}$ types, whereas it spreads out in the B type. Since 
Fig. 5 EDS measurements near the dislocation and in the bulk. a An area of interest for the dislocation area. The dotted square indicate the $0.5 \times 0.5 \mathrm{~nm}^{2}$ boxed area scanned during the measurement. b EDS spectra taken from near the dislocation and in the bulk. c Integrated intensity ratio near the dislocation $\left(I_{\mathrm{dis}}\right)$ to that in the bulk $\left(I_{\text {bulk }}\right)$. The measurements were carried out three times, and the respective cases are shown. The intensities are integrated over the range of $0.48-0.60 \mathrm{keV}, 1.42-1.97 \mathrm{keV}$, and $4.35-4.66 \mathrm{keV}$ for $\mathrm{O}-\mathrm{K}$, $\mathrm{Sr}-\mathrm{L}$, and $\mathrm{Ti}-\mathrm{K}$ lines, respectively (a)

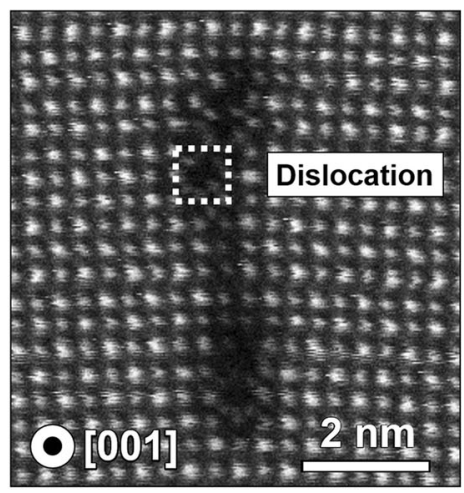

(c)

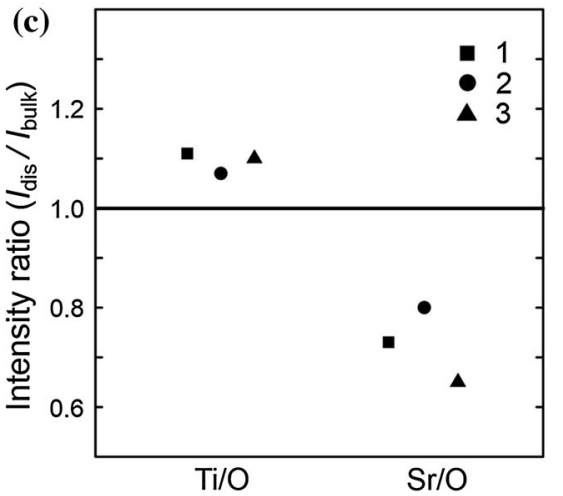

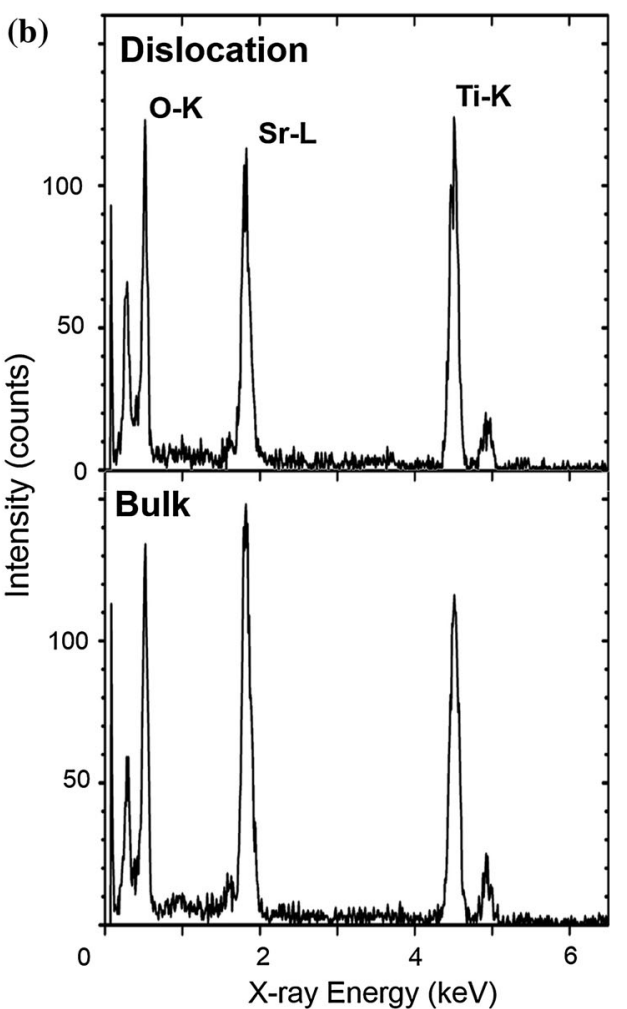

the locations of the Ti-enriched regions correspond with those of extra spots in the STEM images, the origin of the extra spots would be the Ti ion. For further verifying the chemical composition at the Ti-enriched region, EDS spectra were measured. Here, the upper partial in the A type dislocation was chosen as a typical case. The spectra were measured from a $\sim 0.5 \mathrm{~nm} \times 0.5 \mathrm{~nm}$ squared area near the partial dislocation (Fig. 5a) and in the bulk. In the bulk spectrum, the $\mathrm{Sr}-\mathrm{L}$ line has the highest intensity, while the Ti-K line has the highest intensity in the area of the dislocation core (Fig. 5b). The Ti/O ratio is much higher and the $\mathrm{Sr} / \mathrm{O}$ ratio is much lower near dislocation than in the bulk (Fig. 5c), confirming that Ti is enriched and $\mathrm{Sr}$ is depleted near dislocation.

We have identified strain around the dislocations by measuring the distance between some specific columns (Fig. 6a-c), where the column positions are determined by Gaussian peak fitting. First of all, the inter-column distances were measured at the bulk a few $\mathrm{nm}$ from the dislocation. This made it possible to measure the inter-column distances both for a dislocation and in the bulk from the same image, which would help to reduce artifacts. Here, $D_{\text {bulk }}$ is defined as

$D_{\text {bulk }}=\frac{D_{1-1^{\prime}}+D_{2-2^{\prime}}+D_{3-3^{\prime}}+D_{4-4^{\prime}}+D_{5-5^{\prime}}}{5}$,

where $D_{1-1^{\prime}}, D_{2-2^{\prime}}, D_{3-3^{\prime}}, D_{4-4^{\prime}}$, and $D_{5-5^{\prime}}$ are the intercolumn distances between columns 1 and $1^{\prime}, 2$ and $2^{\prime}, 3$ and $3^{\prime}, 4$ and $4^{\prime}$, and 5 and $5^{\prime}$, respectively. Nominal strain perpendicular to the GB $\left(\varepsilon_{110}\right)$ is defined as the ratio of the inter-column distances near dislocation to that in the bulk. In case of the columns $a$ and $A$ near the dislocations in Fig. 6a-c, for example,

$\varepsilon_{110}(\%)=\frac{D_{a-A}-D_{\text {bulk }} \times 4}{D_{\text {bulk }} \times 4} \times 100$,

where $D_{a-A}$ is the inter-column distance between $a$ and $A$. The values of $\varepsilon_{110}$ for the three types of dislocation cases are displayed in Fig. 6d-f. There is high tensile strain near the dislocations, suggesting that it is a cause of the $\mathrm{Ti}$ enrichment and the Sr depletion. Although we have not found any clear relation between the strain values and the size of Ti-enriched region, the highest strain is identified around the largest Ti-enriched area in the B type dislocation.

The exact atomistic structure at the Ti-enriched regions remains unclear. Here, we discuss a possible scenario based on these two points; (i) $\mathrm{Ti}$ is enriched and $\mathrm{Sr}$ is depleted near the dislocations; (ii) It is highly expanded in the dislocation region. (i) The Ti enrichment and the $\mathrm{Sr}$ depletion remind of Ti-richer $\mathrm{Sr}-\mathrm{Ti}-\mathrm{O}$ compounds such as $\mathrm{Sr}_{2} \mathrm{Ti}_{6} \mathrm{O}_{13}$ [17] and $\mathrm{SrTi}_{11} \mathrm{O}_{20}$ [18]. On the other hand, (ii) high tensile strain allows one to imagine that the dislocation region is under less positive or negative pressure compared to the bulk. In the field of high pressure experiments, there is an interesting tendency known as so-called pressure-coordination rule [19]. In the case of some perovskite and the 
Fig. 6 Strain analysis with Gaussian peak fitting. ADF STEM images of a the A type, b the $\mathrm{A}^{\prime}$ type, and $\mathbf{c}$ the $\mathrm{B}$ type dislocations. Cation columns indicated by circles $(a-k, A-K$, $1-5$, and $\left.1^{\prime}-5^{\prime}\right)$ are used for the fitting. Nominal strain estimated for $\mathbf{d}$ the A type, e the $\mathrm{A}^{\prime}$ type, f the B type cases
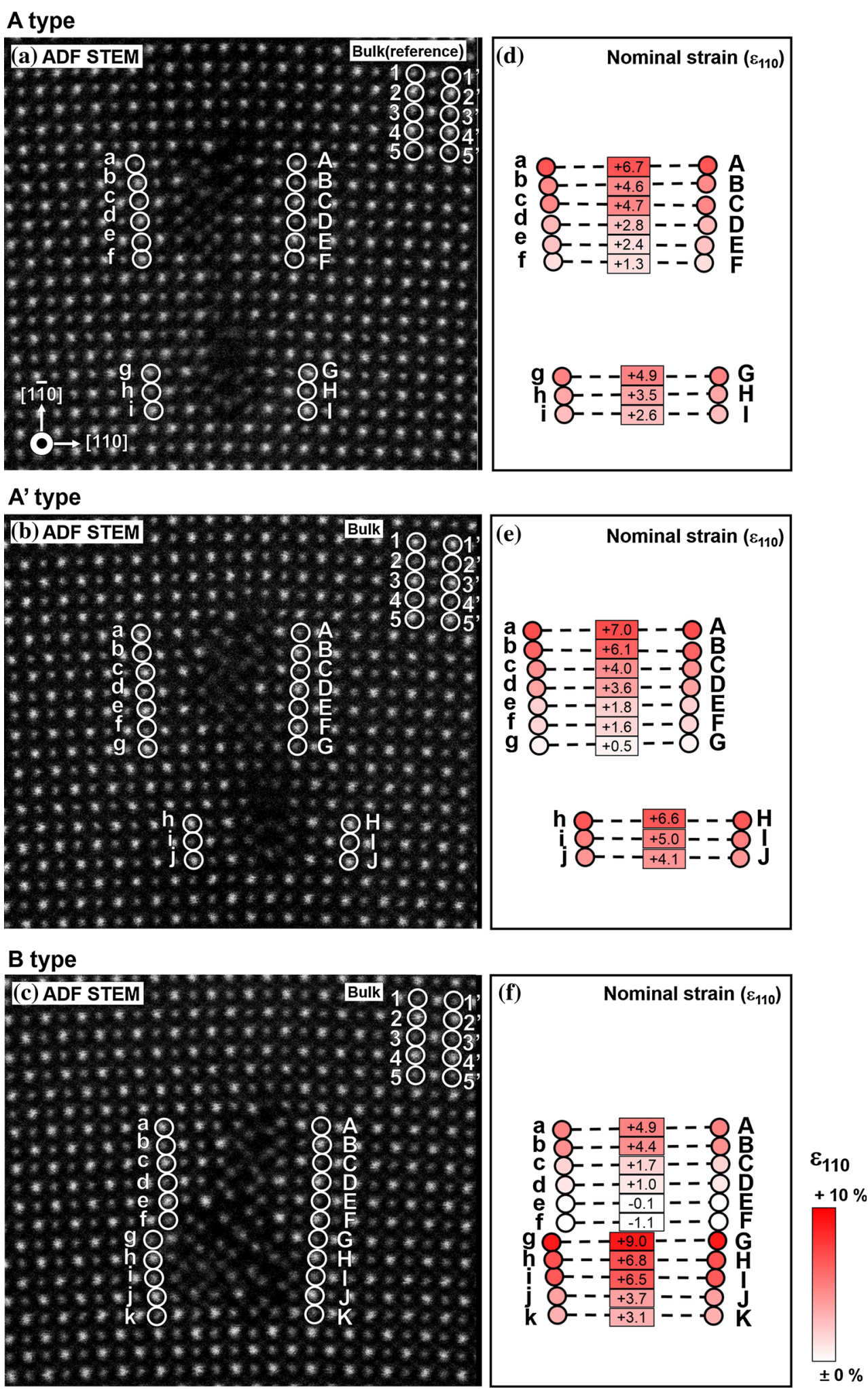

related compounds [20,21], ilmenite crystal structure that is stable under lower pressure transforms into perovskite under higher pressure. Upon the transformation, edgesharing connection of $\mathrm{TiO}_{6}$ octahedra in ilmenite changes into corner sharing in perovskite. As a result, the density of $\mathrm{Ti}$ atoms is locally higher in the low pressure phase (ilmenite) than in the high pressure phase (perovskite). $\mathrm{Sr}_{2} \mathrm{Ti}_{6} \mathrm{O}_{13}$ possesses a different crystal structure from 
(a) Crystal structure of $\mathrm{Sr}_{2} \mathrm{Ti}_{6} \mathrm{O}_{13}$

Ti arrangement (edge-sharing)

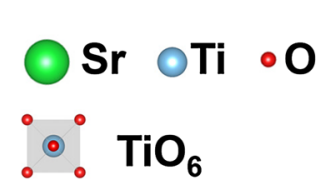

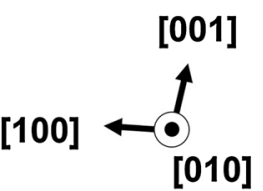

(b) A type

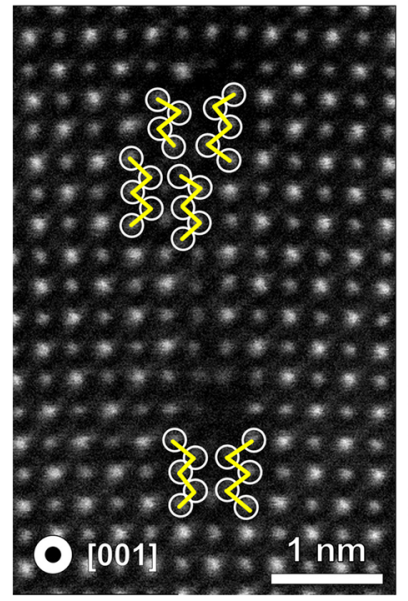

(c) A' type

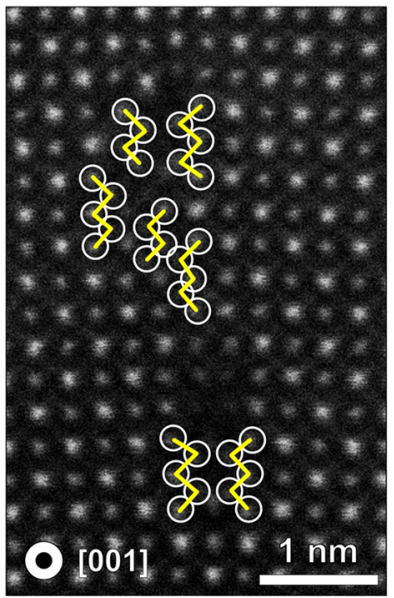

(d) B type

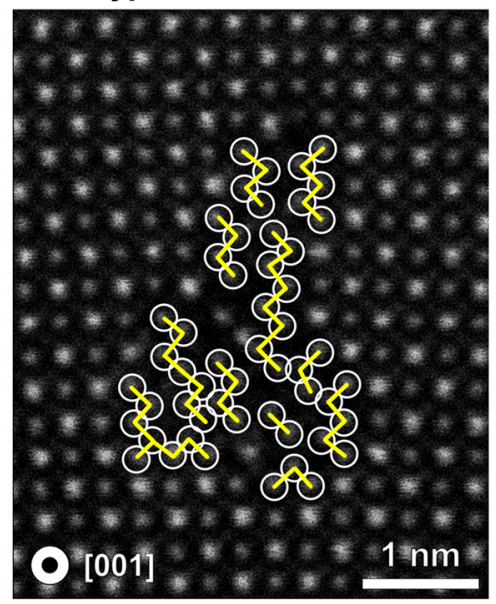

Fig. 7 A structural model at the Ti-enriched region. a Crystal structure of $\mathrm{Sr}_{2} \mathrm{Ti}_{6} \mathrm{O}_{13}$ projected along [010]. The structure is visualized using VESTA [22]. ADF STEM images of $\mathbf{b}$ the A type, $\mathbf{c}$ the $\mathrm{A}^{\prime}$ type, and $\mathbf{d}$ the $\mathrm{B}$ type dislocations. White circles and yellow lines indicate Ti-including columns and their connections (Color figure online) perovskite, with $\mathrm{TiO}_{6}$ octahedra in $\mathrm{Sr}_{2} \mathrm{Ti}_{6} \mathrm{O}_{13}$ connected in an edge-sharing manner (Fig. 7a). When the crystal structure of $\mathrm{Sr}_{2} \mathrm{Ti}_{6} \mathrm{O}_{13}$ is projected along [010], the arrangement of $\mathrm{Ti}$ in the $\mathrm{TiO}_{6}$ octahedra looks similar to that observed at the Ti-enriched regions in the dislocations of Fig. 7b-d. Thus, we suggest that edge-sharing $\mathrm{TiO}_{6}$ octahedra may form a structure in the Ti-enriched regions.

It should be mentioned that some previous studies have suggested Ti enrichment at STO dislocation [6, 7, 23]. For example, Buban et al. [6] proposed a Ti-rich extended core model where Ti-O columns substitute $\mathrm{Sr}$ columns and shift by $a / 2$ [010] to form "nanocolumns of $\mathrm{TiO}_{x}$ " in $a[100]$ edge dislocation. Klie et al. [7] reported that $\mathrm{Ti} / \mathrm{O}$ ratio increases by $18 \%$ at the dislocation core, and Jia et al. [23] reported on an $a$ [001] dislocation with the local atom ratio for Sr:Ti:O of about $0.5: 1.0: 2.3$. These dislocations have different character from that in the present study, implying that the Ti enrichment may be a common phenomenon that occurs in STO dislocation.
Finally, we discuss how the GB in the bicrystal becomes $\mathrm{Ti}$ rich. One potential cause is the surface composition right before bonding. We think that the surface prior to the bonding is stoichiometric, since it was obtained by mechano-chemical polishing. Similar discussion was also reported elsewhere [24]. However, discussion may be complicated because surface structure may change during the heat treatment for bonding [25]. If the crystals are bonded without altering the surface composition, the GB would be kept stoichiometric. Otherwise, the GB may become nonstoichiometric. In addition, heat treatment condition would also affect the GB chemical composition. The present bicrystals were fabricated at relatively low temperature in the air, which is under more oxidized condition [26] as compared with other bicrystal studies. It is reported that [001] high-angle tilt GB of STO becomes Ti richer under oxidizing conditions [27], and similar phenomena would be expected in the low-angle tilt GB case. 


\section{Summary}

$\mathrm{SrTiO}_{3}$ bicrystal with $[001] /(110) 5^{\circ}$ tilt GB was fabricated, and the dislocation structure and composition have been characterized at the atomic scale. STEM-EELS measurement has allowed us to determine that most of the dislocations have Ti-enriched and Sr-depleted regions, with the GB in the crystals being Ti enriched. The extra Ti ions are located at positions unusual for the perovskite crystal, which cannot be understood by a simple structural model. There is a high tensile strain near the dislocations, which would be a cause of the Ti enrichment. We suggest that edge-shared $\mathrm{TiO}_{6}$ octahedra may form in the Ti-enriched regions.

Acknowledgements A part of this work is supported by Grant-inAids for Young Scientist (B) and for Scientific Research on Innovative Areas "Nano Informatics" (25106003) from JSPS, Nihon Sheet Glass Foundation for Materials Science and Engineering, and Kato Foundation for Promotion of Science. The authors thank Prof. T. Mizoguchi, Mr. J. Hoemke, and Mr. A. Fukumoto of the University of Tokyo (UT) and Profs. T. Yamamoto and A. Nakamura of the Nagoya University for useful discussion, proofreading the manuscript, and experimental support. A part of this work was also supported by the Elements Strategy Initiative for Structural Materials (ESISM) and "Nanotechnology Platform" (Project No. 12024046), both sponsored by MEXT, Japan.

Open Access This article is distributed under the terms of the Creative Commons Attribution License which permits any use, distribution, and reproduction in any medium, provided the original author(s) and the source are credited.

\section{References}

1. Nakamura A, Matsunaga K, Tohma J, Yamamoto T, Ikuhara Y (2003) Conducting nanowires in insulating ceramics. Nat Mater $2: 453$

2. Szot K, Speier W, Bihlmayer G, Waser R (2006) Switching the electrical resistance of individual dislocations in single-crystalline $\mathrm{SrTiO}_{3}$. Nat Mater 5:312

3. Ikuhara Y (2009) Nanowire design by dislocation technology. Prog Mater Sci 54:770

4. Zhang Z, Sigle W, Kurtz W, Rühle M (2002) Electronic and atomic structure of a dissociated dislocation in $\mathrm{SrTiO}_{3}$. Phys Rev B $66: 214112$

5. Zhang Z, Sigle W, Rühle M (2002) Atomic and electronic characterization of the $a[100]$ dislocation core in $\mathrm{SrTiO}_{3}$. Phys Rev B 66:094108

6. Buban JP, Chi M, Masiel DJ, Bradley JP, Jiang B, Stahlberg H, Browning ND (2009) Structural variability of edge dislocations in a $\mathrm{SrTiO}_{3}$ low-angle [001] tilt grain boundary. J Mater Res 24:2191

7. Klie RF, Walkosz W, Yang G, Zhao Y (2009) Aberration-corrected Z-contrast imaging of $\mathrm{SrTiO}_{3}$ dislocation cores. J Electron Microsc 58:185
8. Choi SY, Buban JP, Nishi M, Kageyama H, Shibata N, Yamamoto T, Kang SJL, Ikuhara Y (2006) Dislocation structures of low-angle boundaries in $\mathrm{Nb}$-doped $\mathrm{SrTiO}_{3}$ bicrystals. J Mater Sci 41:2621. doi:10.1007/s10853-006-7824-9

9. Ikuhara Y, Nishimura H, Nakamura A, Matsunaga K, Yamamoto $\mathrm{T}$ (2003) Dislocation structures of low-angle and near- $\Sigma 3$ grain boundaries in alumina bicrystals. J Am Ceram Soc 86:595

10. Tochigi E, Shibata N, Nakamura A, Mizoguchi T, Yamamoto T, Ikuhara $\mathrm{Y}$ (2010) Structures of dissociated $<1 \overline{1} 00>$ dislocations and $\left\{\begin{array}{lll}1 \overline{1} 0 & 0\end{array}\right\}$ stacking faults of alumina $\left(\alpha-\mathrm{Al}_{2} \mathrm{O}_{3}\right)$. Acta Mater $58: 208$

11. Sigle W, Sarbu C, Bunner D, Rühle M (2006) Dislocations in plastically deformed $\mathrm{SrTiO}_{3}$. Philos Mag 86:4809

12. Yang KH, Ho NJ, Lu HY (2011) Plastic deformation of $<001>$ single-crystal $\mathrm{SrTiO}_{3}$ by compression at room temperature. J Am Ceram Soc 94:3104

13. Kimoto K, Asaka T, Nagai T, Saito M, Matsui Y, Ishizuka K (2007) Element-selective imaging of atomic columns in a crystal using STEM and EELS. Nature 450:702

14. Lubk A, Rossel MD, Seidel J, Yang SY, Chu YH, Ramesh R, Hÿtch MJ, Snoeck E (2012) Evidence of sharp and diffuse domain walls in $\mathrm{BiFeO}_{3}$ by means of unit-cell-wise strain and polarization maps obtained with high resolution scanning transmission electron microscopy. Phys Rev Lett 109:047601

15. Frank FC (1951) LXXXIII. Crystal dislocations. Elementary concepts and definitions. Philos Mag 42:809

16. Schmidbauer M, Kwasniewski A, Schwarzkopf J (2012) Highprecision absolute lattice parameter determination of $\mathrm{SrTiO}_{3}$, DyScO 3 and $\mathrm{NdGaO}_{3}$ single crystals. Acta Cryst B 68:8

17. Shmachtel J, Müller-Buschbaum H (1980) Oxotitanates with mixed valence, II, about $\mathrm{Sr}_{2} \mathrm{Ti}_{6} \mathrm{O}_{13}$. Z Naturforsch 3:4

18. Hessen B, Sunshine SA, Siegrist T (1991) New reduced ternary titanates from borate fluxes. J Solid State Chem 94:306

19. Manjón FJ, Errandonea D (2008) Pressure-induced structural phase transitions in materials and earth sciences. Phys Status Solidi B 246(1):9

20. Jamieson JC, Lawson AW, Nachtrieb ND (1959) New device for obtaining X-ray diffraction patterns from substances exposed to high pressure. Rev Sci Instrum 30:1016

21. Hirose K, Komabayashi T, Murakami M, Funakoshi K (2001) In situ measurements of the majorite-akimotoite-perovskite phase transition boundaries in $\mathrm{MgSiO}_{3}$. Geophys Res Lett 28:4351

22. Momma K, Izumi F (2011) VESTA 3 for three-dimensional visualization of crystal, volumetric and morphology data. J Appl Crystallogr 44:1272

23. Jia CL, Thust A, Urban K (2005) Atomic-scale analysis of the oxygen configuration at a $\mathrm{SrTiO}_{3}$ dislocation core. Rev Lett 95:225506

24. Szot K, Speier W (1999) Surfaces of reduced and oxidized $\mathrm{SrTiO}_{3}$ from atomic force microscopy. Phys Rev B 60:5909

25. Enterkin JA, Subramanian AK, Russell BC, Castell MR, Poeppelmeier KR, Marks LD (2010) A homologous series of structures on the surface of $\mathrm{SrTiO}_{3}(110)$. Nat Mater 9:245

26. Chan NH, Sharma RK, Smyth DM (1981) Nonstoichiometry in $\mathrm{SrTiO}_{3}$. J Electrochem Soc 128:1762

27. Lee HS, Mizoguchi T, Yamamoto T, Kang SJL, Ikuhara Y (2011) Defect energetics in $\mathrm{SrTiO}_{3}$ symmetric tilt grain boundaries. Phys Rev B 83:104110 